\title{
Numerical Simulation of Circular Synthetic Jets with Asymmetric Forcing Profiles
}

\author{
Ann Marie Thomas* and John P. Abraham
}

School of Engineering, University of St. Thomas, St. Paul, MN, 55105-1079, USA

\begin{abstract}
This paper presents a detailed numerical simulation of the fluid flow characteristics of a synthetic jet. The fluid motion is caused by an oscillating piston within a circular channel which is connected to a larger cavity. The oscillating piston utilized a sawtooth forcing profile, and the solution encompassed the channel geometry, the cavity, and the fluid domain external to the cavity. Momentum fluxes through the orifice and over the exterior of the cavity are calculated. The contribution of the external flow to the induced momentum, by means of the co-flow, is investigated numerically. These findings support previous experimental and computational investigations of the existence of co-flow and its overall contribution to mass and momentum flowrates. The effects of the asymmetric forcing profile on the net and instantaneous momentum fluxes, as well as the co-flow mass flux, are presented. Of particular note, it was found that the shape of the sawtooth profile has a significant impact on direction of momentum transferred to the fluid, however, the absolute magnitudes of this momentum flowrate were found to be negligible compared to the momentum flowing through the orifice.
\end{abstract}

Keywords: Induced co-flow, synthetic jet, momentum flux, propulsion, sawtooth forcing.

\section{INTRODUCTION}

Recently, the viability of synthetic jets as propulsors for underwater vehicles has been demonstrated [1-5]. Inspiration for their use in this application is taken from living creatures that successfully use pulsed jets for their locomotion [6-8]. In a typical synthetic jet, an orifice plate is situated on one wall of a cavity through which fluid is exhausted during a compression stroke. On an expansion stroke, fluid is drawn into the cavity so that throughout an entire cycle, there is no net mass efflux from the cavity. Despite this, synthetic jets are capable of imparting a net momentum to the fluid surrounding the cavity and are also able to promote mixing. In this paper the impact of asymmetric actuation profiles on momentum flux is investigated.

Past investigations demonstrate an evolving understanding of the phenomena which characterize synthetic jet development and the propulsion developed thereby. Experimental work has allowed the clear visualization of jets produced by vibrating motion, acoustic excitation, and other means $[9,10]$. Smith and Glezer performed Schlieren and smoke imaging to identify vortex rings which are ejected from a cavity orifice [11]. Average velocities along the central axis showed the cyclical ingestion and ejection of fluid. In work by Mallinson [12], complementary experimental and numerical studies were performed and comparisons were made. That numerical simulation included both laminar and turbulent models, although the simulations did not extend into the cavity proper. Rather, the presence of a cavity was simulated by an imposed velocity profile at the orifice.

*Address correspondence to this author at the School of Engineering, University of St. Thomas, St. Paul, MN, 55105-1079, USA;

Tel: 651-962-5751; E-mail: apthomas@ stthomas.edu
Calculations have been performed, by Lee and Goldstein on the effect of cavity size and shape on the synthetic jet [13]. In that work, the interaction between a synthetic pulsating jet and a crossflow was investigated. The geometry was planar and the orifice plate was infinite in its extent. More recently, numerical simulations have been conducted looking at the flow induced by synthetic jets on the fluid outside of the jet chamber, upstream of the orifice [14]. That study, inspired by the experimental results of [2] discussed the implications of this induced coflow on the thrust produced by a synthetic jet.

The computations performed in this paper relate to the physical geometry shown in Fig. (1), which is based on information from [2] in which a piston-created flow, in connection with a cylindrical cavity, was used to create synthetic jets and co-flow around the perimeter of the cavity. The piston, modeled here as a moving plate, reciprocates vertically, pulling fluid into the cavity on the upstroke and expelling fluid from the cavity on the downstroke. In addition, fluid surrounds the entire chamber which was submerged within a large volume of water.

One significant difference between the majority of the synthetic jets in the literature and the one presented here is the use of an asymmetrical forcing profile for the piston. Many researchers [15-18] used piezoelectric actuation which allowed for inflow and outflow membrane velocity profiles that were mirror images of one another. Krieg [3] and Mohseni [1], used a plunger with an oscillating velocity profile which was also symmetric with respect to time. Experimental results have been published for a voice-coil actuated synthetic jet with a sawtooth forcing profile $[4,5]$ but to the best of the authors' knowledge, no numerical studies have been done on asymmetricly forced synthetic jets. 
The present calculations will simulate a moving piston, rather than an imposed time-varying velocity profile within the channel which has been more typical of past research. This modification was made to more accurately model the physical situation presented in [2]. The results are focused on the flow patterns at the orifice, within the cavity space, and in the fluid region surrounding the device. Momentum calculations are made to assess the various contributions to the overall momentum transfer to the fluid.

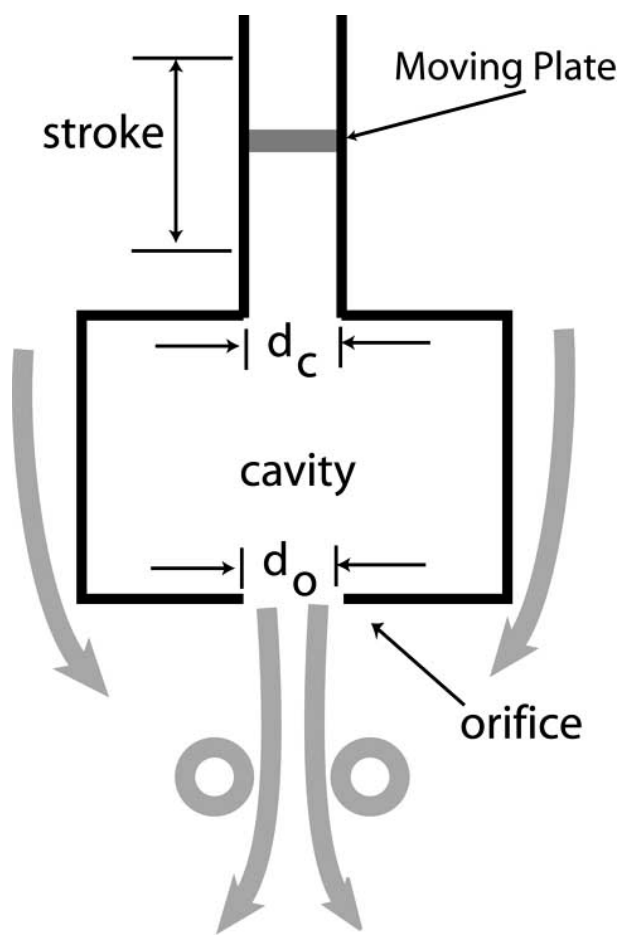

Fig. (1). Schematic diagram of the synthetic jet device.

\section{NUMERICAL MODEL}

\section{Solution Domain}

The physical domain used in the calculations includes the fluid within the channel and cavity and extends both upstream and downstream of the orifice. Both the cavity and channel are circular in shape and the channel is centrally located at the top surface of the cavity. At the top edge of the channel there is a moving plate which reciprocates vertically. The fluid region encompassing the channel-cavity extends laterally and downwards. The size of the solution domain was selected so that the boundary conditions imposed there did not influence the computational results near the investigated cavity.

Arrows in Fig. (1) show the general direction of fluid motion during the outstroke. This motion includes co-flow around the cavity proper, a fluid jet emerging from the orifice, and the vortex rings which are shed from the orifice. The investigations performed in $[2,14,19]$ and the simulations presented here used values of physical parameters shown in Table 1. For the present study, the "co-flow" region refers to the fluid domain outside the cavity, emanating horizontally from the bottom surface of the cavity proper.
Table 1. Synthetic Jet Geometry Used in this Paper

\begin{tabular}{|c|c|c|}
\hline Parameter & Symbol & Value (cm) \\
\hline \hline Cavity Width & $\mathrm{w}_{\mathrm{c}}$ & 7.0 \\
\hline Cavity Height & $\mathrm{h}_{\mathrm{c}}$ & 9.9 \\
\hline Channel Width & $\mathrm{d}_{\mathrm{c}}$ & 2.5 \\
\hline Orifice Width & $\mathrm{d}_{\mathrm{o}}$ & 1.9 \\
\hline
\end{tabular}

\section{Forcing Profile}

As described above, the plate at the top of the chamber is given a velocity profile defined by a sawtooth. Three different sawteeth profiles, as shown in Fig. (2), were used:

- 25/75 Profile: in which the plate is being raised (bringing fluid into the chamber) for $25 \%$ of the period, and being lowered (ejecting fluid from the orifice) for $75 \%$ of the period.

- 50/50 Profile: in which the plate is raised and lowered for equal durations of time.

- 75/25 Profile: in which the plate is being raised (bringing fluid into the chamber) for $75 \%$ of the period, and being lowered (ejecting fluid from the orifice) for $25 \%$ of the period.

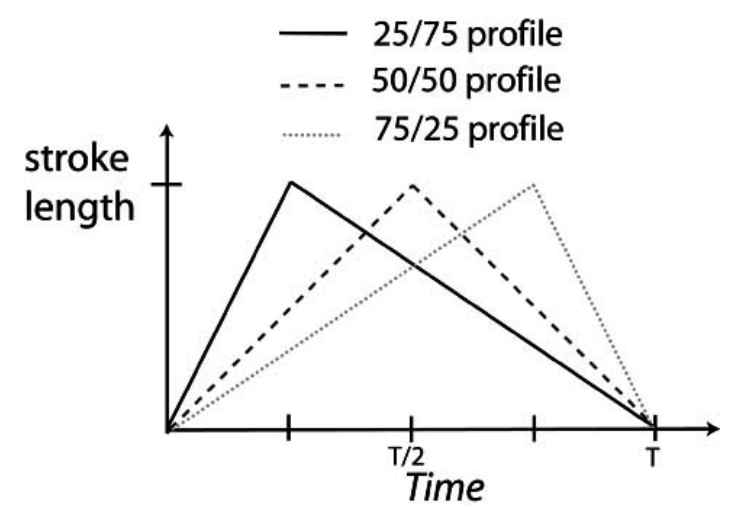

Fig. (2). Sawtooth forcing profiles.

A base case was tested in which the period $(T)$ was one second, and the total stroke of the moving plate was $5 \mathrm{~cm}$. Once this initial case was tested for the three forcing profiles, additional cases were tested with strokes of 2.5, 5.0, and $10 \mathrm{~cm}$ each with periods of $0.5,1$, and 2 seconds in order to assess the impact of stroke length and period on the results.

\section{The Governing Equations}

The flow was modeled as unsteady and incompressible with a timewise deforming mesh that enabled the moving piston. The Navier-Stokes equations for unsteady flow are found in standard fluid flow texts [20,21]. For some of the cases represented by the parameters in Table $\mathbf{1}$ and the forcing profiles in Fig. (2), the flow is turbulent in the channel and at the orifice whereas it is laminar within the cavity itself. In [14], it was shown that the results relevant to this study were independent of whether the turbulence was 
accounted for. Therefore, only results extracted from the laminar solver will be presented here.

The unsteady mass and momentum conservation equations for an incompressible Newtonian fluid are shown in two-dimensional tensor form as

$\frac{\partial u_{i}}{\partial x_{i}}=0$

$\rho\left(u_{i} \frac{\partial u_{j}}{\partial x_{i}}\right)=-\frac{\partial p}{\partial x_{i}}+\mu \frac{\partial^{2} u_{j}}{\partial x_{i}^{2}} \quad j=1,2,3$

where $\mu$ is the dynamic viscosity and $\rho$ is the fluid density.

\section{Initial and Boundary Conditions}

A requirement for the completion of the calculations is that conditions be provided on all boundaries of the fluid domain. At the upper edge of the outer fluid domain, a condition was prescribed which enabled fluid to enter or leave the fluid region (zero gradient was applied to all transport variables). At the rightmost and leftmost boundaries, a wall was employed so that fluid here would be motionless. These boundaries were sufficiently far from the cavity (more than seven times the orifice diameter) so that their impact on the flow was negligible.

At the lower boundary, an exit condition was given with weak closure conditions enforced on the second derivative in the flow direction. This condition allows for flow to exit the domain with minimal impact on the flow near the cavity. Furthermore, the outlet was placed far from the cavity so that its presence did not impact the cavity flow. The location of the outlet, as measured from the bottom of the cavity was 20 times the diameter of the orifice.

The interfaces of solid walls with the fluid were all prescribed as no slip because physically, fluid in contact with a solid wall has no motion.

Initially, at $t=0$, the entire fluid region was at rest. The simulation spanned ten full reciprocating cycles of the piston to ensure that timewise periodic flow was achieved.

\section{The Numerical Method}

The circular geometry of the cavity, channel, and the surrounding fluid body facilitated an axisymmetric simulation. The computations were completed using the CFX11.0 software. In total, more than 80,000 elements were used to resolve the spatial domain. These elements were preferentially deployed in regions were large variations in the transported variables were expected. In addition, thin prismatic elements were deployed along interfaces between the fluid and solid surfaces to ensure proper resolution of the boundary layers. Fig. (3) has been prepared which shows the computational mesh used in the simulation. The figure clearly shows a highly refined region extending throughout the domain and an expanded view showing the wall-adjacent mesh. The utilization of this mesh was based on a rigorous mesh-refinement study so that the solution was independent of element size and deployment. Further details of the mesh refinement are provided in a prior work which utilized the same geometry $[14,19]$.

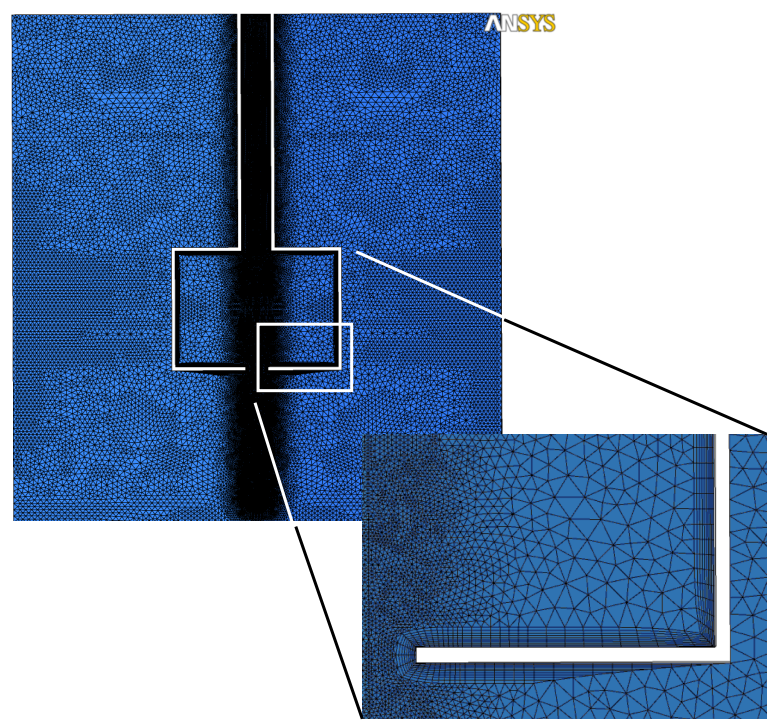

Fig. (3). Computational mesh used in the numerical solution.

The unsteady computations were performed with 100 timesteps for each reciprocating cycle. All timesteps contained 10 internal iterations, each of which was performed using a two-step multi-grid computational algorithm. The time stepping was carried out using a second-order Euler backward scheme. To each time step, a convergence criterion was applied on the root-mean-square value of the residuals of each dependent variable. The convergence criterion that was employed was 0.0001 for all variables. The selection of 100 calculations per period with 10 internal iterations was based on a previous time-step convergence study. During that study, the integration time step and the number of iterations per time step were sequentially reduced until the results were independent of both settings. Independence was based on comparisons of the instantaneous velocity field and on the integrated mass and momentum flowrates through the orifice and co-flow region. Results from the time-step study led to timewise integration steps that were sufficient for all the operating conditions of this study.

Coupling of the velocity-pressure equations was achieved on a non-staggered, collocated grid using the techniques developed by [22] and [23]. The inclusion of pressuresmoothing terms in the mass conservation equation suppresses oscillations which can occur when both the velocity and pressure are evaluated at coincident locations.

The advection term in the momentum equations was evaluated by using the upwind values of the momentum flux, supplemented with an advection-correction term. The correction term reduces the occurrence of numerical diffusion and is of second-order accuracy.

\section{RESULTS AND DISCUSSIONS}

For a finite-sized cavity, momentum is imparted to the fluid in two distinct spatial regions: at the orifice and in the co-flow zone. The results below will be focused on the momentum and mass fluxes in these regions. As synthetic jets have been proposed as a method for mixing fluids, particular interest will be paid to mass flowrates throughout 
the region. The total mass flow through the orifice plane consists of both the flow through the orifice and the flow through the co-flow region. The total mass which passes through the orifice was calculated by numerical integration across the orifice opening at a sequence of times. The actual integration is performed numerically in both space and time and it was found that, as expected, there was no net mass flow through the orifice region so that globally, conservation of mass was achieved.

$m_{\text {orifice }}=\int_{0}^{T}\left(\int_{\text {Orifice Area }} \rho v d A\right) d t=\sum_{i=0}^{t=T}\left[\sum_{\text {Orifice Area }} \rho v_{i} \Delta A_{i}\right] \Delta t=0 \mathrm{~kg}$

The term $v$ signifies the axial component of the fluid velocity. The inner integration (summation) evaluates the instantaneous flow rate through the orifice. The outer integration (summation) calculates the total flow throughout a cycle period. For Eq. (3), the values of the velocity at each elemental area $i$ is orthogonal to both the orifice and the element surface area. Eq. (4) calculates the mass flow through the co-flow region.

$$
m_{\text {co-flow }}=\int_{0}^{T}\left(\int_{\text {Co-flow }} \rho v d A\right) d t=\sum_{t=0}^{t=T}\left[\sum_{C o-\text { flow }} \rho v_{i} \Delta A_{i}\right] \Delta t
$$

Fig. (4) and Table 2 show the total mass which passes through the co-flow region during a single cycle. Fifteen cases were tested, spanning all combinations of the five period/stroke configurations and three sawtooth profiles. As would be expected by conservation of mass and previous work [14, 19], the greatest mass flow is present in the case where the stroke is doubled. However, by using the $75 / 25$ profile, the net mass flow is doubled compared to the 50/50 case and tripled compared to the $25 / 75$ case. It can also be seen that for the double period and double stroke cases, there is a greater sensitivity of co-flow to the time-period split.

Table 2. Net Mass Flow through the co-Flow Region during a Single Up/Down Cycle of the Plate

\begin{tabular}{|c|c|c|c|}
\hline & $\mathbf{2 5 / 7 5}$ & $\mathbf{5 0 / 5 0}$ & $\mathbf{7 5 / 2 5}$ \\
\hline \hline Base Case & $1.29 \times 10^{-3} \mathrm{~kg}$ & $2.07 \times 10^{-3} \mathrm{~kg}$ & $4.46 \times 10^{-3} \mathrm{~kg}$ \\
\hline Double Period & $6.07 \times 10^{-4} \mathrm{~kg}$ & $1.08 \times 10^{-3} \mathrm{~kg}$ & $6.63 \times 10^{-3} \mathrm{~kg}$ \\
\hline Half Period & $2.71 \times 10^{-3} \mathrm{~kg}$ & $4.23 \times 10^{-3} \mathrm{~kg}$ & $9.00 \times 10^{-3} \mathrm{~kg}$ \\
\hline Double Stroke & $7.34 \times 10^{-3} \mathrm{~kg}$ & $1.03 \times 10^{-2} \mathrm{~kg}$ & $2.18 \times 10^{-2} \mathrm{~kg}$ \\
\hline Half Stroke & $5.40 \times 10^{-4} \mathrm{~kg}$ & $1.08 \times 10^{-3} \mathrm{~kg}$ & $2.78 \times 10^{-3} \mathrm{~kg}$ \\
\hline
\end{tabular}

A critical measure of the propulsive capability of synthetic jets is the momentum imparted by the jet to the fluid. For the finite-sized cavity investigated here, momentum was found to be imparted to the fluid in two distinct spatial regions, at the orifice and in the co-flow zone. The momentum flow through the orifice exists even in the absence of a net mass flow. The timewise variation of the orifice momentum flow was determined by spatial integration of the momentum flux at the orifice at a number of successive times, which in turn, were integrated in time. The momentum which has passed through the co-flow region was calculated in a similar fashion. The respective orifice and co- flow momentum flow rates are calculated in Eqs (5) and (6) respectively.

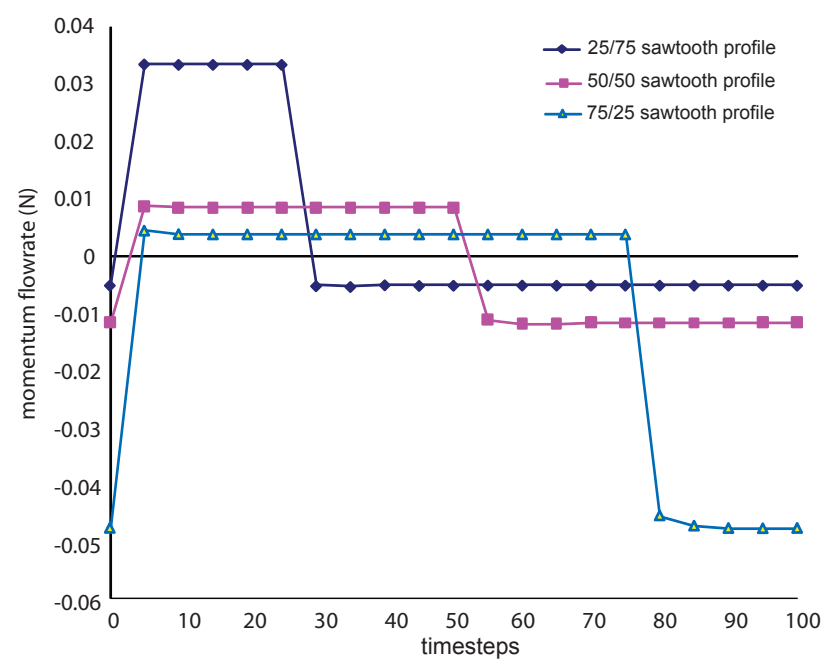

Fig. (4). Single cycle, mass through the co-flow region.

$$
\begin{aligned}
& M_{\text {orifice }}=\int_{0}^{T}\left(\int_{\text {Orifice Area }} \rho v^{2} d A\right) d t=\sum_{t=0}^{t=T}\left[\sum_{\text {Orifice Area }} \rho v_{i}^{2} \Delta A_{i}\right] \Delta t \\
& M_{c o-\text { flow }}=\int_{0}^{T}\left(\int_{\text {Co-flow Area }} \rho v^{2} d A\right) d t=\sum_{t=0}^{t=T}\left[\sum_{\text {Cofflow Area }} \rho v_{i}^{2} \Delta A_{i}\right] \Delta t
\end{aligned}
$$

As would be expected from observing synthetic jets in nature, changing the piston's velocity profile changes the momentum flowrate as a function of time, as well as the overall momentum flowrate per cycle. Table $\mathbf{3}$ shows the time-integration of momentum of fluid passing through the orifice, the co-flow region, and their sum for the base case. Similar results are available for the other reciprocation parameters but are not shown here for sake of brevity. Of particular note are the signs of the flowrates. Note that by changing the profile, the direction of the net momentum transfer can be changed. It can be seen that a $50 / 50$ or $75 / 25$ profile results in a downward thrust (negative) through the orifice, while the $25 / 75$ profile would result in an upward thrust (positive). On the other hand, in every case, it can be seen that the net momentum of the fluid flowing in the coflow region has a negative momentum (directed downwards). Also, a comparison of the orifice momentum flow to that in the co-flow region reveals that in all cases, the momentum flow through the orifice dominates that in the coflow region.

Table 3. Singlecycle Timewise Integrated Momentum Flowrates $(\mathrm{kg} \mathrm{m} / \mathrm{s})$ for the Base Case

\begin{tabular}{|c|c|c|c|}
\hline & $\mathbf{2 5 / 7 5}$ & $\mathbf{5 0 / 5 0}$ & $\mathbf{7 5 / 2 5}$ \\
\hline \hline $\begin{array}{c}\text { Net momentum } \\
\text { transfer (orifice) }\end{array}$ & 0.0896 & -0.0335 & -0.179 \\
\hline $\begin{array}{c}\text { Net momentum } \\
\text { transfer (co-flow } \\
\text { region) }\end{array}$ & $-3.06 \times 10^{-4}$ & $-2.64 \times 10^{-4}$ & $-3.08 \times 10^{-4}$ \\
\hline Total & 0.0892 & -0.0338 & -0.180 \\
\hline
\end{tabular}


Figs. (5) and (6) have been prepared to show the time variation of the momentum flowrates through the orifice and co-flow region respectively throughout one complete cycle (100 time steps).

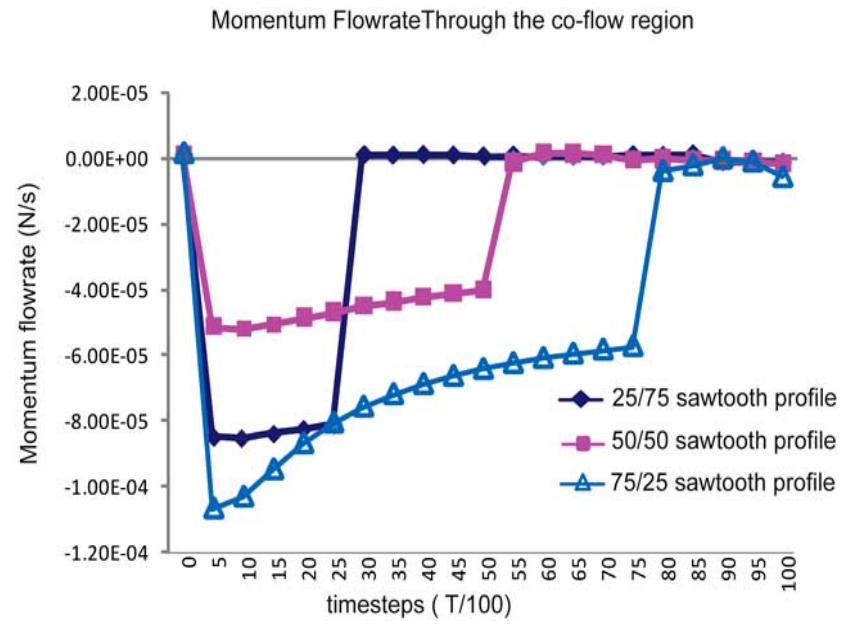

Fig. (5). Momentum flowrate through the orifice with three different forcing profiles for the base case throughout one complete cycle (100 time steps)

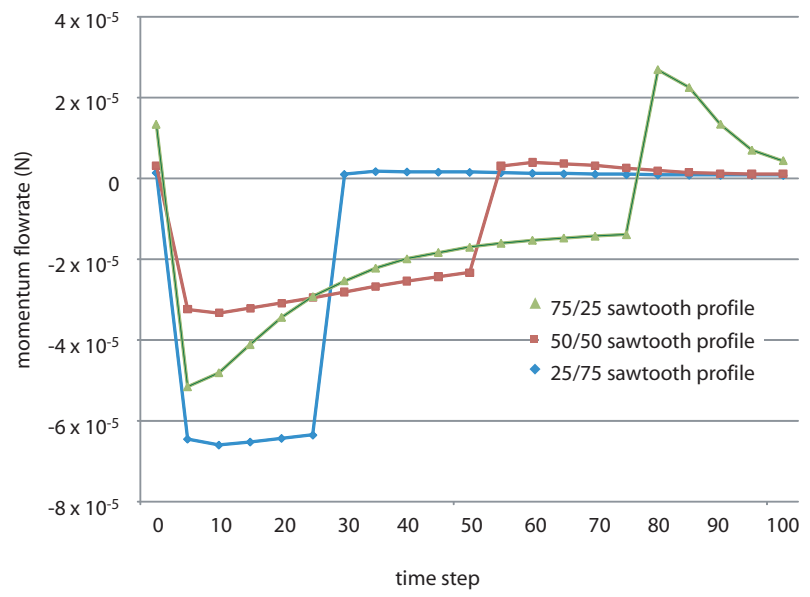

Fig. (6). Momentum flowrate through the co-flow region with three different forcing profiles for the base case.

Finally, Table 4 gives the total net momentum transfer to the fluid considering both the orifice and the co-flow region for the fifteen cases investigated in this study.

An overview of the results of Table 4 show that the momentum transferred to the fluid varies nearly as

$\dot{M}_{\text {orifice }} \sim \frac{\text { stroke length }^{2}}{\text { Period }^{2}}$

Thus, doubling the period results in approximately the same change to momentum as halving the stroke length. As seen in Table 3, $\dot{M}_{\text {orifice }}>>\dot{M}_{c o-f l o w}$, and thus Eq. (7) can be rewritten as
$\dot{M}_{\text {total }} \sim \frac{\text { stroke length }^{2}}{\text { Period }^{2}}$

Table 4. Total Net Momentum Transferred to the Fluid during a Single Up/Down Cycle of the Plate (Units $\operatorname{are~} \mathrm{kg} \mathrm{m} / \mathrm{sec}$ )

\begin{tabular}{|c|c|c|c|}
\hline & $\mathbf{2 5 / 7 5}$ & $\mathbf{5 0 / 5 0}$ & $\mathbf{7 5 / 2 5}$ \\
\hline \hline Base Case & 0.0892 & -0.0338 & -0.180 \\
\hline $\begin{array}{c}\text { Double } \\
\text { Period }\end{array}$ & 0.00292 & -0.00788 & -0.0444 \\
\hline Half Period & 0.352 & -0.141 & -0.726 \\
\hline $\begin{array}{c}\text { Double } \\
\text { Stroke }\end{array}$ & 0.351 & -0.149 & -0.751 \\
\hline Half Stroke & 0.00227 & -0.00735 & -0.0422 \\
\hline
\end{tabular}

This is consistent with the results in a prior investigation which only considered sinusoidal forcing functions [14, 19] and to experimental work carried out previously [2]. This finding is remarkable in that it holds universally for a multitude of forcing functions.

To provide further perspective, Fig. (7) has been prepared. As shown there, the result of changing the period can greatly affect the net momentum transfer to the fluid. Consistent with Eqs. (7) and (8), the difference in single cycle momentum flowrates between the half period and double period cases is nearly a factor of 16 .

As expected, the asymmetric profile significantly impacts the momentum transfer to the fluid. Most dramatic for the half period and double stroke cases, the $75 / 25$ profile results in the doubling of the net momentum transfer compared to the base case. More importantly, however, is the change in direction of the momentum flow. This shows that the same synthetic jet thruster can be used in both "forward" and "reverse" modes as a propellor. A symmetrically forced synthetic jet has only a "forward" mode, significantly constricting the maneuverability of a vehicle using these thrusters.

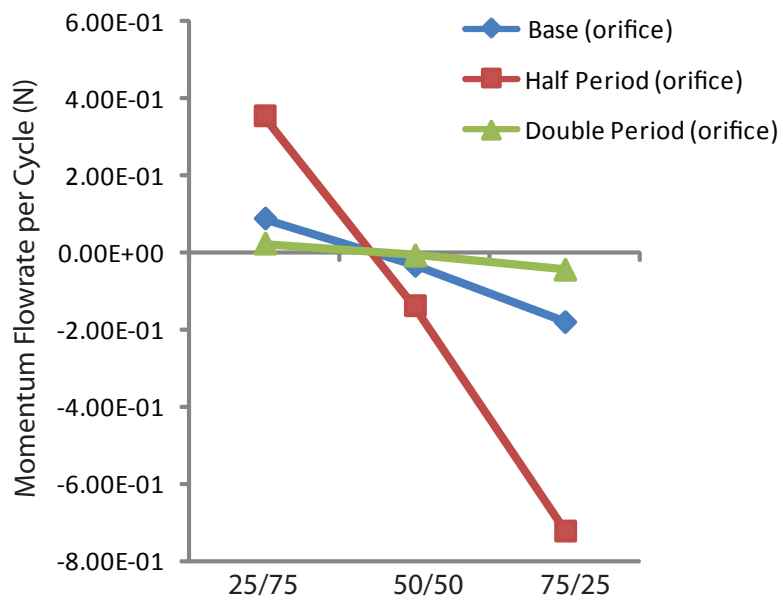

Fig. (7). Single cycle momentum flowrate through the orifice with three different forcing profiles and three different periods. 


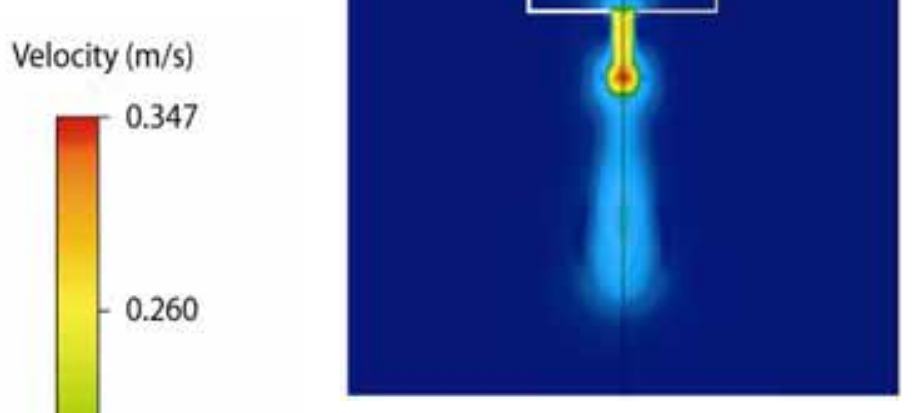

(a)

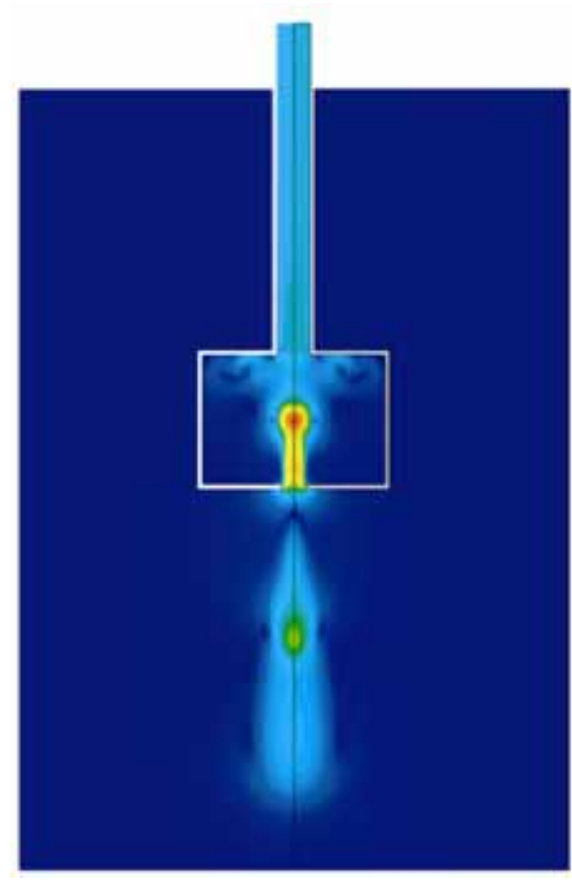

(c)

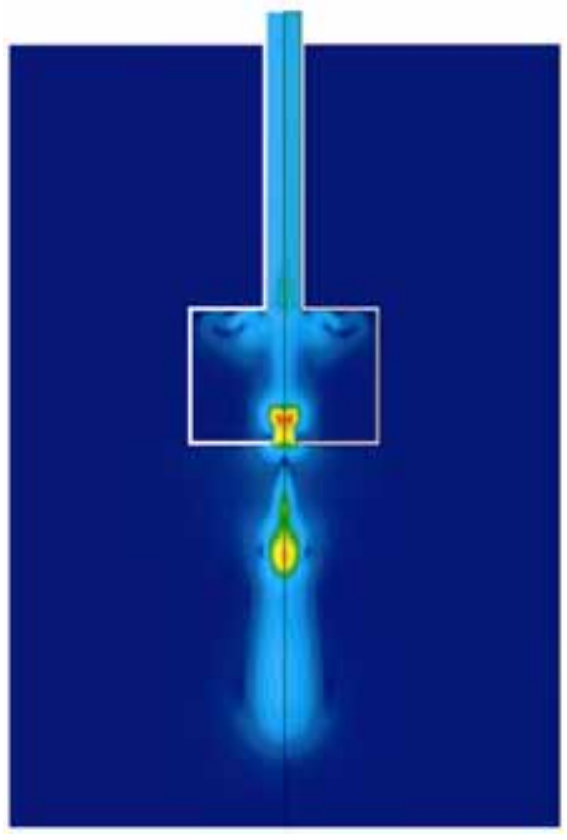

(b)

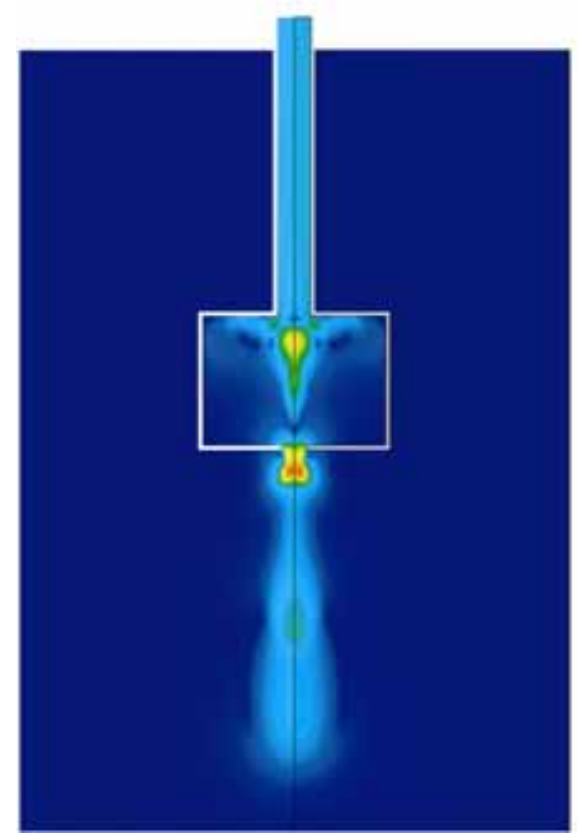

(d)

Fig. (8). Contour plots showing velocity field for the 50/50 base case at $t / T$ values of (a) 0 , (b) 25 , (c) 50 , and (d) 75 . Velocities are keyed to the color contour legend on the left-hand side.

\section{Flow Patterns}

The last presentation of results will display the flow patterns which occur throughout a single period of cyclic motion. The results are extracted from the base case and for a 50/50 sawtooth profile. To facilitate the discussion, Figs. (8a) through (8d) have been prepared which show velocity contours at $t / T$ values of $0,0.25,0.50$, and 0.75 , respectively.
The sequence of figures shows the development of a jetlike slug of fast moving fluid which is being expelled in the (a) part of the figure. The clearly defined slug of fluid is sent into the larger surrounding fluid region. It follows a remnant slug of fluid which was generated during the previous cycle. In (b), the piston is retracting and drawing fluid into the cavity. The slug generated in (a) continues to move away from the orifice and its maximum velocity is decreasing, as 
evidenced by the yellow color of that fluid. In (c), the ingested jet is shown extending deeply into the cavity and the piston is at its uppermost position. Finally, in (d), the fluid is again being ejected from the cavity as the piston is in a downward trajectory. During the motion, complex recirculation patterns and evident which are similar to those previously identified in $[14,19]$.

\section{CONCLUDING REMARKS}

Numerical simulation of the fluid motion induced by an asymmetrically forced, reciprocating piston within a circular channel has been carried out. The focus of the simulations was on the flow patterns near the orifice plate which comprises one wall of the cavity and in the region surrounding the cavity. Results were provided for the mass and momentum flows through the orifice and in the region of fluid surrounding the cavity. It was found that both the direction and magnitude of a synthetic jet's momentum flow can be altered by using an asymmetric forcing profile. As synthetic jets are increasingly being discussed as a means of underwater vehicle propulsion, the ability to produce a wider range of thrust magnitudes and direction from a single jet expands the range of applications for which these jets might be used.

\section{NOMENCLATURE}

$\begin{aligned} \mathrm{A} & =\text { area }\left[\mathrm{m}^{2}\right] \\ \mathrm{d}_{\mathrm{c}} & =\text { channel width }[\mathrm{cm}] \\ \mathrm{d}_{\mathrm{o}} & =\text { orifice width }[\mathrm{cm}] \\ \mathrm{h}_{\mathrm{c}} & =\text { cavity height }[\mathrm{cm}] \\ \mathrm{i}, \mathrm{j} & =\text { tensor indices } \\ \mathrm{m} & =\text { mass }[\mathrm{kg}] \\ \mathrm{M} & =\text { momentum }[\mathrm{kg} \mathrm{m} / \mathrm{s}] \\ \mathrm{p} & =\text { pressure }[\mathrm{Pa}] \\ \mathrm{t} & =\text { time }[\mathrm{s}] \\ \mathrm{T} & =\text { period }[\mathrm{s}] \\ \mathrm{u}_{\mathrm{i}} & =\text { local velocity }[\mathrm{m} / \mathrm{s}] \\ \mathrm{x} & =\text { coordinate direction }[\mathrm{m}] \\ \mathrm{w}_{\mathrm{c}} & =\text { cavity width }[\mathrm{cm}]\end{aligned}$

[11] B. Smith, and A. Glezer, "The formation and evolution of synthetic jets", Phys. Fluids, vol. 10, pp. 2281-2297, 1998.

[12] S. Mallinson, J. Reizes, and G. Hong, "An experimental and numerical study of synthetic jet flow", Aeronaut. J., vol. 105, pp. 41-49, 2001.

[13] C. Lee, and D. Goldstein, "Two-dimensional synthetic jet simulation", AIAA J., vol. 40, no. 3, pp. 510-516, 2002.

[14] J. Abraham, and A. Thomas, "Induced co-flow and laminar-toturbulent transition with synthetic jets", Comput. Fluids, vol. 38, pp. 1011-1017, 2009.

[15] A. Crook, A.M. Sadiri, and N.J. Wood, "The development and implementation of synthetic jets for the control of separated flow", 30th Appl Aerodyn Conf, Norfolk, VA, 1999.

[16] S. Mallinson, G. Hong, and J. Reizes, "Some Characteristics of Synthetic Jets", $30^{\text {th }}$ AIAA Fluid Dyn Conf, Norfolk, VA, 1999.

[17] F.-J. Chen, C. Yao, G.B. Beeler, R.G Bryant, and R.L. Fox, "Development of synthetic jet actuators for active flow control at NASA Langley", AIAA Paper 2000-2405, 2000.

[18] B. Smith and A. Glezer, "Vectoring and small-scale motions effected in free shear flows using synthetic jet actuators", AIAA Paper 97-0213, 1997.

[19] Abraham, and A. Thomas, "Numerical simulation of induced coflow and laminar-to-turbulent transition associated with syntheticjets", Proc Flucome 2007, Tallahassee, FL, Sept. 16-19, 2007.

[20] H. Schlichting, and K. Gersten, Boundary Layer Theory, $8^{\text {th }}$ ed., Berlin: Springer Verlag, 2000.

[21] F. White, Viscous Fluid Flow, $3^{\text {rd }}$ ed., New York: McGraw Hill, 2005.

[22] C. Rhie, and W. Chow, "A numerical study of the turbulent flow past an isolated airfoil with trailing edge separation", AIAA J., vol. 21, 1982, pp. 170-183.

[23] S. Majumdar. "Role of underrelaxation in momentum interpolation for calculation of flow with nonstaggered grids", Numer. Heat Transf., vol. 13, pp. 125-132, 1998. 